\title{
Preditores de qualidade de vida em pacientes renais crônicos
}

\author{
Life quality predictors in renal chronic patients
}

\author{
Tânia RUDNICKI ${ }^{1}$
}

\begin{abstract}
Resumo
Neste trabalho foram avaliados os preditores do índice de qualidade de vida nas suas duas dimensões - satisfação e importância - em pacientes renais crônicos em tratamento de hemodiálise. A comparação entre os critérios do índice demonstrou uma média significativamente mais elevada para a importância, bem como uma menor dispersão das respostas. A análise de regressão múltipla mostrou associação entre estressores e tratamento de hemodiálise. O apoio social, no que se refere ao tamanho da rede e ao nível de satisfação com o apoio percebido, relaciona-se ao índice de qualidade de vida nas dimensões satisfação e importância.
\end{abstract}

Unitermos: apoio social; paciente renal crônico; psicologia da saúde; qualidade de vida.

\begin{abstract}
Life quality Index predictors were evaluated in a renal chronic hemodialysis patients in regard to their two dimensions, Satisfaction and Importance. The Index criteria comparison showed a significant greater average for the Importancelevels, as well as a small answers dispersion. The multiple regression analysis demonstrated an association between stressors and hemodialysis treatment. Social support, in regard to the patients'net and the Satisfaction level for the perceived support, relates to Satisfaction and Importance dimensions of the Life Quality index.

Uniterms: social support; chronic renal patient; health care psychology; quality of life.
\end{abstract}

Ao longo da trajetória vital as pessoas passam por numerosos acontecimentos, alguns deles geradores de grandes impactos emocionais. O mais crítico, provavelmente, é a questão da própria morte. Aos pacientes renais crônicos em tratamento de hemodiálise, que podem ser considerados como um grupo à parte entre os doentes crônicos, essa proximidade suscita um amplo leque de atitudes e emoções.

As enfermidades crônicas não se definem pela sua aparente ou real gravidade, mas sim por não terem cura ou serem de duração muito prolongada. Abrangem nomeadamente doenças que conduzem, num prazo mais longo ou mais curto, à morte. Adaptar-se às características da doença renal constitui um processo extremamente complexo, com inúmeras implicações e repercussões de variadas ordens, sendo necessário valorizar a qualidade dessa sobrevida.

Alguns dos sintomas apresentados pelo enfermo renal em tratamento de hemodiálise podem traduzir-se em diversos graus de limitação física e condições de trabalho. Além de um problema de saúde é também

\section{$\boldsymbol{\nabla} \boldsymbol{\nabla} \boldsymbol{V}$}

1 Universidade Luterana do Brasil, Curso de Graduação e Pós-Graduação em Psicologia. Av. Farroupilha, 8001, São José, 92425-900, Canoas, RS, Brasil. E-mail: $<$ taniarudd@terra.com.br>. 
um problema social e econômico (Lindqvist, Carlsson \& Sjoden, 2000; Mok \& Tam, 2001; Parsons \& Harrys, 1997).

Estudos de conceitos como qualidade de vida, apoio social, estresse e estressores são realizados junto a esse grupo de enfermos com a finalidade de conhecer suas necessidades (Anderson \& Ferrans, 1997; Elgueta, Manãlich \& Saffie, 1994; Ferrans \& Powers, 1982).

Pesquisas sobre a ligação estresse-doença têm mostrado os efeitos fisiológicos do estresse em doentes renais crônicos submetidos a vários estressores, dentre eles a própria doença, o procedimento de hemodiálise, o regime de restrição dietética e hídrica, a ingestão crônica de medicamento, e, em vários casos, a expectativa frente à realização de um transplante (Holahan \& Moos, 1985; Klang, Bjorvell, Berglung, Sundsted \& Cline 1998; Krediet, 2001; Machado \& Car, 2003; Steptoe, 2000).

Estudo realizado por Elgueta et al. (1994) aponta o efeito do estresse tanto nos comportamentos específicos relacionados à saúde quanto nas mudanças comportamentais, e que a limitação das atividades físicas deve-se à anormalidade endócrina, considerada como estressor fisiológico, e a disfunção social, às dificuldades em relação ao papel de doente. Além disso, o enfermo se depara com alterações psicológicas decorrentes do próprio tratamento devido às múltiplas perdas: a liberdade, a expectativa de vida, a incerteza quanto ao futuro, a troca de papel social na família, o emprego, o medo de ficar sozinho (Baldree, Murphy \& Powers, 1982; Daneker, Kimmel, Ranich \& Peterson, 2001; Franke, Reimer, Philipp \& Heemann, 2003).

O controle sobre os agentes estressores possui um importante efeito sobre a saúde, sendo a adaptação à doença um processo complexo que depende de inúmeros fatores (Franke et al., 2003; Heiwe, Clyne \& Dahlgren, 2003). Dentre os pacientes renais crônicos em tratamento hemodialítico, um dos fatores que se vê mais afetado por essa complexidade de aspectos é a adesão ao tratamento. As pesquisas que buscam identificar diferenças individuais correlacionadas a 글 $\quad$ resultados de adesão nessa população são limitadas. Kimmel et al. (1998) afirmam que 30\% a 50\% dos pacientes em hemodiálise não aderem à dieta, à restrição hídrica e/ou ao regime medicamentoso.

O trabalho de Anderson e Ferrans (1997), cuja estrutura conceitual foi dirigida pelo modelo de qualidade de vida de Ferrans, descreve que em pacientes com uma doença crônica, os resultados são especialmente importantes. Dificuldades nas áreas social, familiar e de trabalho, descrédito ou precária aceitação da doença são fatores revelados nessa pesquisa, como significantes rupturas em muitas áreas da vida.

Quanto ao apoio social, seu conceito pode ser interpretado de várias formas; a maior parte dos investigadores o considera como um conceito multidimensional (Cobb, 1976; Barrera, 1986; Sarason \& Sarason, 1987; Sarason, Shearin \& Pierce, 1987). Apesar da diversidade de definições apresentadas, possuem todas, como foco comum, a capacidade de auxílio e os processos dos sistemas de relações sociais (Gottlieb, 1988; Krediet, 2001).

Dentre os conceitos existentes, aquele defendido pelos autores do Questionário de Apoio Social (SSQ), Sarason, Levine, Bashan e Sarason (1983), é bastante claro. Ele pretende ver um indivíduo comprometendose com um leque de relações, cada uma delas diferindo em qualidade e profundidade de preocupação. No entanto o efeito principal da comunicação desses sentimentos não é, por si só, proteger, mas gerar confiança.

O apoio social pode ser entendido como a qualidade do suporte emocional disponível a partir das relações estabelecidas nas redes sociais, e sua presença ou ausência afeta diferentemente a saúde dos indivíduos. A natureza dessa relação explicaria o porquê de indivíduos com rede de apoio na forma de família, amigos e companheiros freqüentemente terem melhores condições de saúde física e mental, tendo em vista os recursos emocionais e/ou materiais que dela obtêm (Sarason et al., 1983; Sarason et al., 1990; Heiwe et al., 2003).

Nesse campo do conhecimento, questões ligadas ao doente renal crônico obtiveram avanços significativos. Neste estudo, considera-se a definição de qualidade de vida proposta pelo modelo de Ferrans (1990), por sua abrangência e pela adequação aos objetivos.

A qualidade de vida pode ser entendida, dessa forma, como a percepção individual de bem-estar, variando da satisfação à insatisfação em relação aos domínios da vida que lhe são importantes. 
Os aspectos enfocados no presente estudo são: identificar repercussões do tratamento hemodialítico relacionadas ao apoio social e aos estressores vinculados à qualidade de vida do paciente crônico; analisar se as variáveis biodemográficas e socioeconômicas influenciam em sua qualidade de vida; verificar em que medida o apoio social se relaciona com sua qualidade de vida; buscar a relação, se existente, entre estressores e qualidade de vida, além de identificar o perfil discriminante entre homens e mulheres, em tratamento hemodialítico, a partir das variáveis em estudo. Especificamente busca-se levantar os preditores da qualidade de vida desses pacientes a partir da escala criada por Ferrans e Powers (1985), que defendem a importância do construto, refletindo sua complexidade.

Tendo em vista os aspectos aqui levantados, busca-se responder qual a relação entre as variáveis, apoio social, estressores percebidos e características biodemográficas com a qualidade de vida de pacientes renais crônicos em tratamento de hemodiálise.

\section{Método}

\section{Participantes}

A população de pacientes renais crônicos em tratamento de hemodiálise, na cidade de Porto Alegre, RS, é de mais de duas mil pessoas, de ambos os sexos, e com idade variável, cujo tratamento é pago pelo Sistema Único de Saúde (SUS) (Sociedade Brasileira de Nefrologia, 2004). A amostra foi selecionada a partir de avaliação médico-clínica, efetivada por médicos nefrologistas responsáveis por duas clínicas de hemodiálise, localizadas na capital e Grande Porto Alegre, envolvendo 168 pacientes com idade mínima de 19 e máxima de 82 anos que aceitaram participar do trabalho, assinando o Termo de Consentimento. No critério de escolha, foram excluídos os pacientes que, pela avaliação médica, não apresentaram condições físicas suficientes para responder aos instrumentos.

\section{Instrumentos}

Os instrumentos utilizados foram: Questionário de Dados Sociodemográficos, Escala de Apoio Social; Índice de Qualidade de vida e Estressores relacionados ao tratamento de hemodiálise.
Índice de qualidade de vida (Quality of Life IndexQOL): criado como medida de avaliação da percepção sobre a qualidade de vida (QOL) de pacientes em tratamento dialítico. A versão para diálise consiste de 68 itens divididos em duas seções; a primeira avalia o quanto de satisfação a pessoa apresenta em 34 aspectos de sua vida; a segunda aponta a importância desses mesmos aspectos. A consistência interna da versão diálise do QOL aponta um Alpha de 0,90 e 0,93 para escala total; e Alpha de 0,87, 0,82, 0,90 e 0,77 para as escalas de saúde/funcionamento, social/econômico, psicológico/espiritual e familiar, respectivamente, elaboradas por Ferrans e Powers $(1985,1993)$.

Escala de Apoio Social (Social Support Questionnaire [SSQN-SSQS]): instrumento psicometricamente satisfatório que lida com as relações entre apoio social e lócus de controle de um lado; persistência e interferência cognitiva sob condições de frustração, de outro (Sarason et al., 1983). Foi escolhida a aplicação da escala reduzida pelos próprios autores, tendo em vista as condições dos participantes da pesquisa (SSQ6), com itens selecionados da totalidade para aplicação em pessoas com alguma enfermidade física. Enquanto no SSQ total o fator Alpha de consistência interna ficou entre 0,97 e 0,98, para SSQN e 0,96 a 0,97 para SSQS, na forma reduzida, essa consistência foi de 0,90 a 0,93, tanto para N como para S (Sarason et al., 1987). Quanto ao número de pessoas listadas (N), o estudo dos criadores do instrumento aponta uma média de 87,34. A média para a escala S (satisfação) foi de 114, 26, enquanto a correlação entre $\mathrm{N}$ e S foi de 0,43.

Estressores em Hemodiálise (Hemodialysis Stressors Scale): a Escala criada por Baldree et al. (1982) inclui vários estressores presentes no tratamento hemodialítico. Conforme avaliação de propriedades psicométricas do instrumento (Alpha=0,86), os estressores mais comuns, por ordem decrescente, são a perda da função corporal, a limitação das atividades físicas, o tratamento prolongado, o cansaço, as cãibras musculares, o fator econômico, as mudanças na aparência física, a restrição hídrica, a fistula artereovenosa, as náuseas e os vômitos. O instrumento tem 29 itens ( 6 fatores psicológicos e 23 psicossociais) que medem as respostas dos pacientes.

\section{Procedimentos}

Foi formalizado pedido para aplicação dos instrumentos da pesquisa em clínicas de hemodiálise 
no Rio Grande do Sul; após autorização de Comitê de Ética em Pesquisa, formalizou-se o contato com os locais disponíveis. A partir de avaliação médica positiva, tempo de início do tratamento estimado em noventa dias, idade mínima de 18 anos, os pacientes que apresentaram condições de discernimento para aceitar ou não participar da pesquisa foram convidados. A partir da aquiescência da direção das clínicas, dos médicos responsáveis e do próprio paciente, foi efetivada a aplicação dos instrumentos. Dessa forma foi, então, assinado o Termo de Consentimento Livre e Esclarecido juntamente com a autorização do médico responsável e pontuados por escrito os objetivos do estudo.

Os instrumentos foram aplicados durante a sessão de hemodiálise, sendo cada um deles entregue em folhas previamente preparadas para facilitar a visualização dos itens e, por conseguinte, da resposta. Cada questão foi lida pela pesquisadora que registrava a resposta no protocolo de pesquisa. Os procedimentos asseguraram a confidencialidade, a privacidade, a proteção à imagem e a não estigmatização, garantindo que as informações não seriam utilizadas em prejuízo das pessoas.

Os dados foram levantados e pontuados em um banco de dados especificamente elaborado para este estudo. Inicialmente receberam uma análise descritiva que buscou identificar o grupo pesquisado em suas variáveis sociodemográficas. A seguir, por meio da análise de regressão múltipla, foram identificados os preditores de qualidade de vida, sendo, então, realizadas duas análises de regressão, uma para cada dimensão de qualidade de vida avaliada: satisfação e importância.

\section{Resultados}

Os dados foram inicialmente analisados pelo teste " $\mathrm{t}$ " de Student a fim de comparar as médias dos grupos (homens e mulheres), verificando a diferença entre eles (Tabela 1).

Para a escala QOL e suas subescalas, verifica-se pelo teste "t", ao nível de significância de 1\%, que os homens apresentam uma média significativamente maior do que as mulheres. Também se verifica que não há diferença entre a média do índice de apoio social em relação ao gênero, através do teste " $\mathrm{t}$ " $(p=0,400)$. Para a escala de estressores e suas subescalas, verifica-se pelo teste "t", ao nível de significância de 5\%, que os homens apresentam uma média significativamente menor do que as mulheres.

A fim de verificar quais fatores podem predizer o índice de qualidade de vida nas duas dimensões do construto, utilizou-se a análise de regressão múltipla com as seguintes variáveis dependentes: idade, atividade remunerada, plano de saúde, tamanho da rede, índice de satisfação com o apoio social, índice de estressores fisiológicos e índice de estressores psicológicos.

Para uma melhor compreensão da distribuição da variável dependente (VD) índice de qualidade de vida, apresenta-se, na Tabela 2, as médias dos sujeitos da amostra de acordo com os percentis a partir dos instrumentos de pesquisa.

A Tabela 3 mostra o modelo final para qualidade de vida obtida através da análise de regressão múltipla.

Tabela 1. Médias, diferenças em termos de gênero no que diz respeito a homens e mulheres em tratamento de hemodiálise - escalas de qualidade de vida, apoio social e estressores, utilizando teste " $t$ ".

\begin{tabular}{|c|c|c|c|c|c|c|c|}
\hline \multirow{3}{*}{ Escala /subescala } & & & \multicolumn{4}{|c|}{ Gênero } & \multirow{3}{*}{$p$} \\
\hline & \multicolumn{2}{|r|}{ Geral } & \multicolumn{2}{|c|}{ Masculino } & \multicolumn{2}{|c|}{ Feminino } & \\
\hline & Média & Desvio-padrão & Média & Desvio-padrão & Média & Desvio-padrão & \\
\hline Qualidade de vida & 21,23 & 4,07 & 22,22 & 3,59 & 20,07 & 4,30 & 0,004 \\
\hline Saúde e funcionamento & 19,74 & 4,77 & 20,73 & 4,33 & 18,60 & 5,01 & 0,005 \\
\hline Social e econômica & 20,47 & 4,46 & 21,36 & 4,42 & 19,44 & 4,32 & 0,005 \\
\hline Psicológica/espiritual & 23,01 & 5,00 & 24,04 & 4,25 & 21,81 & 5,55 & 0,003 \\
\hline Familiar & 25,02 & 5,93 & 26,32 & 4,69 & 23,53 & 6,82 & 0,001 \\
\hline Apoio social & 5,09 & 1,28 & 5,02 & 1,35 & 5,18 & 1,20 & 0,400 \\
\hline Estressores & 2,63 & 0,80 & 2,49 & 0,78 & 2,78 & 0,79 & 0,019 \\
\hline Fisiológicos & 2,73 & 0,82 & 2,61 & 0,84 & 2,88 & 0,78 & 0,033 \\
\hline Psicológicos & 2,47 & 0,90 & 2,33 & 0,85 & 2,64 & 0,93 & 0,026 \\
\hline
\end{tabular}


As três variáveis que entraram sucessivamente na análise como preditoras do índice de QOL explicaram 29\% da variância. As variâncias encontradas apresentam neste estudo um nível satisfatório. Quando se tem uma amostra de 168 sujeitos, a variância explicada tende a diminuir na medida em que aumenta a amostra.

As variáveis, índice de estressores fisiológicos, tamanho da rede e plano de saúde explicam 29,2\% da variação do índice de qualidade de vida. Desse percentual de explicação, 70,1\% correspondem ao índice de estressores fisiológicos (Tabela 4).

No grupo estudado, as mulheres, que apresentaram maior número de estressores, apontam a interferência no trabalho, o cansaço e o tratamento prolongado - todos estressores fisiológicos - como os três mais freqüentes. Do grupo de homens, a limitação das atividades físicas, as mudanças na aparência física e a perda da função corporal/física foram os mais freqüentes.

Tabela 2. Fatores preditores do índice de qualidade de vida - Análise de regressão múltipla com as seguintes variáveis dependentes: idade, atividade remunerada, plano de saúde, tamanho da rede, índice de satisfação com o apoio social, índice de estressores fisiológicos e índice de estressores psicológicos.

\begin{tabular}{|c|c|c|c|c|c|c|c|c|c|}
\hline \multirow[b]{2}{*}{ Percentil } & \multicolumn{5}{|c|}{ Qualidade de vida } & \multirow[b]{2}{*}{ Apoio Social } & \multicolumn{3}{|c|}{ Estressores } \\
\hline & Total & $\begin{array}{c}\text { Saúde e } \\
\text { funcionamento }\end{array}$ & $\begin{array}{c}\text { Social e } \\
\text { econômico }\end{array}$ & $\begin{array}{l}\text { Psicológica/ } \\
\text { espiritual }\end{array}$ & Familiar & & Total & Fisiológico & Psicológico \\
\hline 5 & 13,83 & 11,20 & 12,33 & 11,92 & 11,18 & 1,74 & 1,45 & 1,38 & 1,17 \\
\hline 10 & 15,44 & 12,46 & 13,95 & 15,91 & 15,45 & 2,67 & 1,60 & 1,62 & 1,33 \\
\hline 15 & 16,23 & 14,24 & 16,12 & 18,88 & 20,43 & 4,33 & 1,75 & 1,88 & 1,50 \\
\hline 20 & 18,13 & 15,67 & 16,99 & 19,71 & 22,08 & 4,83 & 1,93 & 2,04 & 1,67 \\
\hline 25 & 18,81 & 16,60 & 17,47 & 20,57 & 22,78 & 5,00 & 2,03 & 2,13 & 1,83 \\
\hline 30 & 19,57 & 17,42 & 18,51 & 21,39 & 24,00 & 5,00 & 2,17 & 2,29 & 1,92 \\
\hline 35 & 20,14 & 18,20 & 19,51 & 22,31 & 25,00 & 5,03 & 2,28 & 2,41 & 2,00 \\
\hline 40 & 20,86 & 18,74 & 20,06 & 22,66 & 25,50 & 5,17 & 2,43 & 2,53 & 2,09 \\
\hline 45 & 21,40 & 19,61 & 20,67 & 23,14 & 26,63 & 5,33 & 2,51 & 2,59 & 2,17 \\
\hline 50 & 21,85 & 20,29 & 21,29 & 23,75 & 27,00 & 5,50 & 2,59 & 2,65 & 2,30 \\
\hline 55 & 22,07 & 21,17 & 22,06 & 24,00 & 27,00 & 5,67 & 2,63 & 2,83 & 2,52 \\
\hline 60 & 22,93 & 21,44 & 22,50 & 24,67 & 28,05 & 5,83 & 2,76 & 2,94 & 2,67 \\
\hline 65 & 23,21 & 22,13 & 22,67 & 25,35 & 28,50 & 6,00 & 2,83 & 3,00 & 2,83 \\
\hline 70 & 23,63 & 22,80 & 23,25 & 25,81 & 28,50 & 6,00 & 2,97 & 3,06 & 2,91 \\
\hline 75 & 24,08 & 23,17 & 23,58 & 26,39 & 30,00 & 6,00 & 3,11 & 3,29 & 3,00 \\
\hline 80 & 24,82 & 24,00 & 24,00 & 27,71 & 30,00 & 6,00 & 3,23 & 3,42 & 3,25 \\
\hline 85 & 25,26 & 24,73 & 24,70 & 28,29 & 30,00 & 6,00 & 3,50 & 3,59 & 3,42 \\
\hline 90 & 26,16 & 25,73 & 25,33 & 28,80 & 30,00 & 6,00 & 3,70 & 3,83 & 3,73 \\
\hline 95 & 27,05 & 26,38 & 26,32 & 29,42 & 30,00 & 6,00 & 4,05 & 4,25 & 4,13 \\
\hline
\end{tabular}

Tabela 3. Índice de qualidade de vida, satisfação e importância: variáveis que entraram na equação de regressão com seus respectivos pesos.

\begin{tabular}{|c|c|c|c|c|c|}
\hline \multicolumn{6}{|l|}{ Índice de qualidade vida - satisfação } \\
\hline Variável & $\mathrm{B}$ & SEB & Beta & $\mathrm{t}$ & Sig. $\mathrm{t}$ \\
\hline Estressores relacionados à hemodiálise & $-0,420$ & 0,066 & $-0,456$ & $-6,364$ & 0,000 \\
\hline Apoio social - tamanho da rede & $-0,309$ & 0,076 & $-0,292$ & $-4,088$ & 0,000 \\
\hline Sexo & $-0,344$ & 0,115 & $-0,215$ & $-2,987$ & 0,003 \\
\hline Plano de saúde & $-0,409$ & 0,146 & $-0,200$ & $-2,800$ & 0,006 \\
\hline \multicolumn{6}{|l|}{ Índice de qualidade vida - importância } \\
\hline Variável & $\mathrm{B}$ & $\mathrm{B}$ & Beta & $\mathrm{t}$ & Sig. $\mathrm{t}$ \\
\hline Estressores relacionados à hemodiálise & $-0,196$ & 0,052 & $-0,312$ & $-3,778$ & 0,000 \\
\hline Idade & $-1,171 \mathrm{E}-02$ & 0,003 & $-0,295$ & $-3,525$ & 0,001 \\
\hline Apoio social - satisfação & $9,913 \mathrm{E}-02$ & 0,034 & 0,239 & 2,916 & 0,004 \\
\hline Plano de saúde & $-0,273$ & 0,113 & $-0,195$ & $-2,406$ & 0,018 \\
\hline
\end{tabular}


Tabela 4. Análise de regressão múltipla com as variáveis significativas.

\begin{tabular}{lccc}
\hline Variáveis independentes significativas & $\beta$ padronizado & $p$ & $I m p a c t o(\%)$ \\
\hline Índice de estressores fisiológicos & $-0,432$ & $<0,001$ & 70,1 \\
Tamanho da rede & 0,226 & 0,001 & 19,2 \\
Plano de saúde & 0,168 & 0,015 & 29,2 \\
\hline
\end{tabular}

*Variável dependente: índice de qualidade devida.

Tabela 5. Médias (M) e desvio-padrão (DP). Dimensões da escala de qualidade de vida - satisfação e importância.

\begin{tabular}{lcc}
\hline Índice de qualidade de vida & $M$ & DP \\
\hline Satisfação & 4,52 & 0,78 \\
Importância & 5,37 & 0,57 \\
\hline
\end{tabular}

1: muito insatisfeito; 2: moderadamente insatisfeito; 3: pouco insatisfeito; 4: pouco satisfeito; 5: moderadamente satisfeito; 6: muito satisfeito.

Observa-se que, em média, os 168 sujeitos da amostra revelam estar de pouco a moderadamente satisfeitos (dimensão de QOL) com os aspectos relacionados à sua qualidade de vida (Tabela 5). Com relação à importância (dimensão de QOL) que atribuem a esses aspectos, encontra-se a opção moderadamente importante. Na comparação entre essas duas dimensões de qualidade de vida, verifica-se uma média sensivelmente mais elevada em termos da importância, bem como uma menor dispersão das respostas.

\section{Discussão}

No que tange aos aspectos biodemográficos, encontra-se diferença em termos de sexo, variável apontada como preditora do índice de qualidade de vida. A variável sexo se apresenta como preditor de qualidade de vida - satisfação, indicando melhor índice para os homens. Uma possibilidade bastante objetiva que se contempla é a expectativa de vida, maior para as mulheres (Kimmel, Emont, Newman, Danko \& Moss, 2003; Kimmel \& Patel, 2003).

Duas variáveis entram como preditoras de ambas as dimensões: os estressores relacionados ao tratamento de hemodiálise que ocupam o primeiro lugar em importância nas duas dimensões e a posse de plano de saúde, que ocupa o último (Baldree et al., 1982; Kimmel et al., 1998).

Apesar da diferença do poder explicativo dessas variáveis nas duas dimensões de qualidade de vida, pode-se buscar compreendê-las sob um mesmo foco. Ambas estão estreitamente ligadas às circunstâncias de vida relacionadas à doença renal crônica e, mais especificamente, ao tratamento em geral (Levenson \& Glocheski, 2000; Pierratos, 2004; Tovbin et al., 2003; Wasserfallen et al., 2004).

Questões sobre fatores estressores abarcam aspectos fisiológicos e psicológicos presentes no tratamento, geralmente aspectos bastante comuns, mas que alteram a rotina diária do enfermo. O tratamento da doença renal crônica não se restringe apenas à hemodiálise, mas inclui uma série de cuidados na alimentação e restrição de ingesta hídrica, além das repercussões psicológicas pela realização periódica das sessões de hemodiálise. A própria doença e seu tratamento ocasionam sintomas que alteram de forma radical o funcionamento global da pessoa (Kimmel, 2000; Mok\&Tam, 2001).

No grupo estudado, as mulheres apresentaram maior número de estressores, apontando a interferência no trabalho, o cansaço e o tratamento prolongado entre os mais freqüentes estressores, todos eles fisiológicos. No grupo de homens, a limitação das atividades, as mudanças na aparência física e a perda da função corporal foram os mais freqüentes.

Os resultados do estudo de Baldree et al. (1982) indicam que os estressores psicossociais têm um impacto equivalente aos estressores fisiológicos. A restrição hídrica atingiu o maior nível como estressor, além das cãibras musculares e da fadiga. É nesse sentido que se identifica a contribuição de o paciente possuir um plano de saúde e de esse servir como preditor de melhores índices de qualidade de vida em geral. Ter um plano de saúde representa segurança de atendimento, além de possibilitar a minimização de intercorrências inevitáveis no caso de enfermidade renal. Durante uma doença é necessário ampliar a capacidade de adaptação do enfermo na busca de proteção e suporte (Courts, 2000; Levenson \& Glocheski, 2000). 
No presente estudo, o fato de possuir ou não plano de saúde prediz ambas as dimensões da escala de qualidade de vida - satisfação e importância. Nas duas, a direção da variável é a mesma, ou seja, os sujeitos que possuem planos de saúde apresentam melhor índice de qualidade de vida.

Embora a hemodiálise seja totalmente subsidiada pelo Sistema Único de Saúde, a comorbidade, os exames e as consultas necessários ao bem-estar da pessoa não estão previstos com a mesma qualidade. Outro dado importante refere-se à origem da doença renal. Na grande maioria dos casos, esse quadro clínico deriva de uma outra enfermidade, entre as mais comuns estão a hipertensão arterial sistêmica (HAS) e a diabetemellitus (DM) (Cormier-Daigle \& Stewart, 1997; Van Manen et al., 2003). Outrossim, a HAS aparece em 40\% dos respondentes e a DM em 46\%, confirmando, dessa forma, as informações da literatura especializada.

O apoio social também se apresenta como preditor para qualidade de vida em suas duas dimensões, porém com diferente conotação. Para a dimensão de satisfação, se insere, no modelo de regressão, como a segunda variável preditora em termos de importância, representando a satisfação do sujeito com o tamanho da sua rede social de apoio. A mudança de comportamento e a modalidade de enfrentamento devem objetivar a busca por um melhor nível de qualidade de vida (Kimmel, 2000; Kimmel et al., 2003; Krediet, 2001).

É preciso considerar a vulnerabilidade real do doente renal crônico e o sentimento de desamparo na situação do tratamento. É nesse ponto que a satisfação com o apoio social importa, em termos bastante objetivos ou estruturais, referindo-se ao tamanho da rede. É a amplitude da rede que proporciona maior segurança e portanto, satisfação ao enfermo, posto que ele conta com mais recursos, mesmo que não dimensione corretamente sua importância. Não há dúvidas quanto ao processo de apoio dos familiares se constituir uma experiência positiva (Courts, 2000; Daneker et al., 2001).

Já no que tange ao que realmente o paciente considera importante em termos de apoio social percebido é a sua satisfação com o mesmo. Pode-se pensar que esses dados são incongruentes, se observar que a satisfação entra como o terceiro preditor de qualidade de vida - importância - e aparece de forma distinta na dimensão satisfação. Entretanto, quando se fala de importância, pensa-se em algo avaliado pela pessoa, não importando tanto o tamanho da rede, mas o quanto as pessoas ou os recursos de apoio estão cumprindo seu papel na percepção do indivíduo. A importância remete, portanto, ao aspecto mais funcional do apoio social, levando-se em conta a satisfação do sujeito quanto ao que ele percebe como apoio. A natureza dessa relação explicaria por que indivíduos com rede de apoio na forma de família, amigos e companheiros freqüentemente têm melhores condições de saúde física e mental, tendo em vista os recursos emocionais e/ou materiais que obtêm (Sarason et al., 1983; Tardy, 1985).

Quanto aos aspectos biodemográficos, são encontradas diferenças entre as dimensões: sexo prediz índice de qualidade de vida - satisfação, e idade prediz índice de qualidade de vida - importância (Kimmel et al., 2003; Kimmel \& Patel, 2003). O estudo apresenta média de idade de 52 anos. A variável sexo constitui o terceiro preditor de qualidade de vida - satisfação, indicando melhor índice para os homens. Uma possibilidade bastante objetiva contemplada é a expectativa de vida que é maior para as mulheres. Considerando-se que o instrumento de pesquisa sobre qualidade de vida abarca quatro dimensões: saúde/funcionamento, aspectos socioeconômicos, aspectos psicológicos/espirituais e aspectos familiares, pode-se formular a hipótese de que as mulheres podem sofrer as repercussões da perda do companheiro, o que ocorre com menor freqüência entre os homens. Isso está diretamente relacionado à dimensão de aspectos familiares, tendo o casamento importante papel. Além disso, é uma característica da mulher ser a cuidadora. No casamento é a mulher que, principalmente, assume esse papel, não abandonando o marido no decorrer da enfermidade, o que já não ocorre quando a mulher está enferma (Tovbin et al., 2004; Wu et al., 2004).

Dentre os preditores de qualidade de vida importância, encontra-se a idade como o segundo preditor, indicando melhores índices em pessoas mais jovens. Na juventude pode-se encontrar uma certa ilusão de invulnerabilidade ou mesmo um distanciamento das reais ameaças da doença, o que diminui com o aumento da idade. Além disso, a juventude permite uma maior 
inserção em, pelo menos, um campo a mais de acordo com as dimensões do instrumento, ou seja, a dimensão socioeconômica. As pessoas com mais idade têm prejuízo nesse campo, uma vez que o avanço da idade é empecilho no mercado de trabalho, assim como nas opções sociais. Isso se agrava quando a idade vem acompanhada de uma doença crônica especialmente limitante em função de seu tratamento, como é o caso da doença renal. A atividade é um grande fator de saúde e bem-estar psicológico e, nesse sentido, os jovens têm mais possibilidades (Cieza, Estremadoyro \& Tenório, 1995).

Observa-se a tendência a apresentar menor função física e baixo engajamento às atividades sociais. Sintomas como dor, juntamente com fatores psicossociais e espirituais, são fundamentais determinantes de qualidade de vida desses pacientes. Os determinantes clínicos de mortalidade consistem em idade avançada, presença de diabetes mellitus e outras condições co-mórbidas (Kimmel, 2000; Kimmel et al., 2003).

Conhecendo os preditores de qualidade de vida em geral, pode-se pensar no grande peso que deriva da própria doença renal e de seu tratamento. Também alguns aspectos biodemográficos, quando avaliados com um pano de fundo social, revelam-se como importantes preditores. É justamente esse enfoque que encaminha o estudo para o campo social, baseado no apoio percebido, uma vez que fornece possibilidade de atuação preventiva a fim de promover uma melhor qualidade de vida para essa população. É o campo no qual podem ocorrer mudanças, desde as referentes à própria percepção dos indivíduos em termos do apoio social, até aquelas que envolvem contextos mais amplos, como os culturais e sociais.

\section{Conclusão}

A comparação entre os critérios do índice de qualidade de vida demonstrou uma média significativamente mais elevada para a importância, bem como uma menor dispersão das respostas. A análise de regressão múltipla apontou associação entre estressores e tratamento de hemodiálise, e o apoio social, no que se refere ao tamanho da rede e ao nível de satisfação com o apoio percebido, relaciona-se ao índice nas suas dimensões satisfação e importância.
Este estudo identificou repercussões do tratamento hemodialítico relacionadas ao apoio social e aos estressores vinculados à qualidade de vida do paciente renal crônico; os homens apresentam melhores índices de qualidade de vida do que as mulheres que, por sua vez, encontram-se mais estressadas. Portanto os homens têm melhores condições, uma vez que quanto maior o índice de qualidade de vida, melhores condições possui para enfrentar os estressores relacionados à doença.

O tratamento da doença renal crônica inclui uma série de cuidados, além das repercussões psicológicas da realização periódica das sessões de hemodiálise. A própria doença e seu tratamento ocasionam sintomas que alteram de forma radical o funcionamento global da pessoa, por isso a importância de o profissional da saúde conhecer mais de perto esse paciente. Assim, o estudo revela a importância de manter ou melhorar a qualidade de vida, sendo essa a mais importante meta terapêutica para muitos enfermos com uma doença crônica, como a insuficiência renal terminal.

\section{Referências}

Anderson, J. S. R. N., \& Ferrans, C. E. (1997). The Quality of Life of Persons with Chronic Fatigue Syndrome. Journal of Nervous Mental Disease, 185 (6), 359-367.

Baldree, K. S., Murphy, S. P., \& Powers, M.J. (1982). Stress identification and coping patterns in patients on hemodialysis. Nursing Research, 31 (2), 107-112.

Barrera, M. (1986). Distinctions between social support concepts, measures and models. American Journal of Community Psychology, 14 (4), 413-445.

Cieza, J., Estremadoyro, L., \& Tenorio A. (1995). Influencia de la hemodiálisis sobre la capacidad laboral de pacientes en hemodiálisis crónica intermitente. Revista Medica Heredia, 6 (1), 27-32.

Cobb, S. (1976). Social support as a moderator of life stress. Psychosomatic Medicine, 38 (5), 300-313.

Cormier-Daigle, M., \& Stewart, J. (1997). Support and coping of male hemodialysis-dependent patients. International Journal of Nursing, 34 (6), 420-430.

Courts, N. F. (2000). Psychosocial adjustment of patients on home hemodialysis and their dialysis partners. Clinical Nursing Research, 9 (2), 177-191.

Daneker, B., Kimmel, P. L., Ranich, T., \& Peterson, R. A. (2001). Depression and marital dissatisfaction in patients with end-stage renal disease and their spouses. American Journal of Kidney Disease, 38 (4), 839-846. 
Elgueta, L., Manãlich, J., \& Saffie, A. (1994). Chronic Hemodialysis: program for evaluation the quality of life patient over 60 years old. Revista Médica de Chile, 122 (6), 679-685.

Ferrans, C. E., \& Powers, M. J. (1982). Psychometric assessment of quality of life index. Nursing Research, 15 (3), 29-38.

Ferrans, C. E., \& Powers, M. J. (1985). Quality of Life Index: development and psychometric properties. Advertising Nursing Science, $8(1), 15-24$

Ferrans, C. E. (1990). Quality of life: conceptual issues. Seminars in Oncology Nursing, 6 (2), 248-254.

Ferrans, C. E., \& Powers, M. J. (1993). Quality of life of hemodialysis patients. ANNA Journal, 20 (5), 575-581.

Franke, G. H., Reimer, J., Philipp, T., \& Heemann, U. (2003). Aspects of quality of life through end-stage renal disease. Quality of Life Research, 12 (2), 103-115.

Gottlieb, B. H. (1988). Social networks and social support (Vol.4). Beverly Hills: Sage Publications.

Heiwe, S., Clyne, N., \& Dahlgren, M. A. (2003). Living with chronic renal failures: patients experiences of the physical and functional capacity. Physiotherapy Research International, 8 (4), 167-177.

Holahan, C. S., \& Moos, R. H. (1985). Life stress and health personality, coping and family support in stress resistance. Journal of Personality and Social Psychology, 49 (3), 739-747.

Kimmel, P., Petersen, R. A., Wheis, K. L, Simmens, S. J., Alleyne, S, Cruz, I, Veis, J. H. (1998). Psychosocial factors, behavioral compliance and survival in urban hemodialysis patients. Kidney International, 54 (1), 245-254.

Kimmel, P. L. (2000). Psychosocial factors in adult end-stage renal disease patients treated with hemodialysis: correlates and outcomes. American Journal of Kidney Disease, 35 (4 Suppl), 132-140.

Kimmel, P. L., Emont, S. L., Newmann, J. M., Danko, H., \& Moss, A. H. (2003). ESRD patient quality of life: sintoms, spiritual beliefs, psychosocial factors, and ethnicity. American Journal Kidney Disease, 42 (4), 713-721.

Kimmel, P. L., \& Patel, S. S. (2003). Psychosocial issues in women with renal disease. Advertising renal Replace Therapy, 10 (1), 61-70.

Klang, B., Bjorvell, H. R. N., Berglung, J., Sundsted, R. N., \& Cline, N. (1998). Pre-dialysis patient education: effects on functioning and well-being in uraemic patients. Journal of Advertising Nursery, 28 (1), 36-44.

Krediet, R. T. (2001). Changes in employment status in endstage renal disease patients during their first year of dialysis. Peritoneal Dialysis International, 21 (6), 595-601.

Levenson, J. L., \& Glocheski, S. (2000). Doença renal terminal. In A. Stoudemire. Fatores psicológicos afetando condições médicas. Porto Alegre: Artmed.

Lindqvist, R, Carlsson, M., \& Sjoden, P. O. (2000). Perceived consequences of being a renal failure patient. Nephrology Nursing, 27 (3), 291-7.

Machado, L. R., \& Car, M. R. (2003). A dialética da vida diária de pacientes com insuficiência renal crônica: o inevitável e o casual. Revista da Escola de Enfermagem da USP, 37 (3), 2735.

Mok, E., \&Tam, B. (2001). Stressors and coping methods among chronic hemodialysis patients in Hong Kong. Journal of Clinic Nursing, 10 (4), 503-511.

Parsons, D. S., \& Harrys, D. C. (1997). A Review of Life in Chronic Renal Failure. Pharmacoeconomics, 12 (2), 140160.

Pierratos, A. (2004). New approaches to hemodialysis. Annual Revue Medicine, 55 (3), 179-189.

Sarason, B. R., Levine, H. M., Bashan, R. B., \& Sarason, I. G. (1983). Assessing social support: the social support questionnaire. Journal of Personality and Social Psychology, 44 (1), 121-137.

Sarason, I. G., \& Sarason, B. R. (1986). Experimentally provided social support (1986). Journal of Personality and Social Psychology, 50 (6), 1222-25

Sarason, B. R., Shearin, E. D., \& Pierce, G. R. (1987). Interrelations of social support measures: theoretical and practical implications. Journal of Personality and Social Psychology, 52 (4), 813-832.

Sarason, B. R. Sarason, I. G., \& Pierce, G. R. (1990). Social support: na international view. New York: John Willey.

Steptoe, A. (2000). Stress, social support and cardiovascular activity over workday. International Journal of Psychophysiology, 37 (3), 299-380.

Sociedade Brasileira de Nefrologia (2004). Capítulos de nefrologia - diálise. Acessado em junho 18, 2004, disponível em: http://www.sbn.br

Tardy, C. H. (1985). Social support measurement. American Journal of Community Psychology, 13 (3), 27-36.

Tovbin, D., Gidron, Y., Jean, T., Granovsky, R., \& Shneider, A. (2003). Relative importance and individualized quality of life of hemodialysis patients. Quality of Life Research, 12 (6), 709-717.

Van Manen, J. G., Korevaar, J. C., Dekker, F.W., Reuselaars, M. C., Boeschot, E. W., Vasquez, I., Valderrabano, F., Jofre, R., Fort, J., Lopez-Gomez, J.M., Moreno, F., \& Sans-Guajardo, D. (2003). Psychosocial factors and quality of life in Young hemodialysis patients with low co morbidity. Journal of Nephrology, 16 (6), 886-894.

Wasserfallen, J. B., Halabi, G., Saudan, P., Perneger, T., Feldman, H. I., Martin, P. Y., \& Wauters, J. P. (2004). Quality of life on chronic dialysis: comparison between haemodialysis and peritoneal dialysis. Nephrology and Dialysis Transplantation, 5 (3), 234-236.

Wu, A. W., Fink, N. E., Marsh-Manzi, J. U., Meyer, K. B.; Finkelstein, F.O., Chapman, M. M., \& Powe, N. R. (2004). Changes in quality of life during hemodialysis treatment: generic and disease specific measures. Quality of Life Research, 22 (8),1002-1006.

Recebido em: 13/10/2005

Versão final reapresentada em: 26/1/2007

Aprovada em: 7/2/2007 
